\title{
Infección por SARS-CoV-2 y tuberculosis pulmonar: análisis de la situación en el Perú
}

\author{
SARS-CoV-2 infection and pulmonary \\ tuberculosis: an analysis of the situation in Peru
}

\author{
Infecção por SARS-CoV-2 e tuberculose pulmonar: \\ análise da situação no Peru
}

Pool Aguilar-León 1
Jose Cotrina-Castañeda 1
Ernesto Zavala-Flores 1

doi: 10.1590/0102-311X00094520
La infección por SARS CoV-2, denominada COVID-19, se inició y propagó desde China hacia el resto del orbe con resultados nocivos para la salud pública y economía mundial. En Latinoamérica, Brasil fue el primer país en informar un caso en febrero de 2020 con una posterior y gradual expansión continental 1; heterogénea en temporalidad y número de casos, y homogénea en términos de desenlace socioeconómico. A mediados de agosto, Sudamérica viene registrando más de 5 millones de casos y cerca de 200 mil decesos 2. En el Perú, tras la confirmación del primer caso a inicios de marzo 3, las medidas adoptadas por el gobierno buscaron mitigar el contagio masivo y la afectación de poblaciones vulnerables, a través de la imposición de una cuarentena temprana, cierre de fronteras, restricción de viajes nacionales e internacionales, distanciamiento social, otorgamiento de bono de apoyo social a poblaciones en pobreza y extrema pobreza, retiro adelantado de fondos de sistemas de pensiones privados y cierre de escuelas y universidades, en un afán por evitar el colapso de los servicios de salud; de por sí debilitados 4 .

Sin embargo, pese a las medidas de contención adoptadas, se viene constatando el desborde y colapso de los sistemas de salud en territorio nacional. Perú presenta un elevado número de contagiados, superando los 550 mil casos y ubicándose como la segunda nación de Latinoamérica, después de Brasil, con el mayor número de infectados. De los casos confirmados, cerca de 15 mil peruanos se encuentran en servicios de hospitalización y más de 500 reciben ventilación mecánica 5 . Sobre la mortalidad, se vienen notificando más de 25 mil fallecidos 2; afectando desproporcionadamente a la población adulta mayor 6 y colocando al país dentro de las diez naciones con mayor cantidad de decesos a causa del nuevo coronavirus 7 . La magnitud de la pandemia es comparada con la observada en los puntos críticos europeos 8 , con número de infectados y fallecidos aumentando rápidamente y que sigue una heterogénea distribución regional, en relación a la temporalidad con un impacto inicial en la capital Lima, regiones del oriente y norte, y una sucesiva afectación del sur; sin distinción de clima, geografía o altitud 4 .

Los motivos por los cuales el Perú viene fracasando en su lucha contra el COVID-19 incluyen una falta de infraestructura y logística adecuada de los sistemas sanitarios a nivel nacional, una centralización del plan de respuesta en hospitales de la capital Lima, un bajo presupuesto inicial para enfrentar la pandemia que incluye suministros médicos insuficientes y el poco compromiso por parte de la población para acatar las medidas de contención impuestas por el gobierno; en parte sustentada por

\author{
1 Hospital Nacional \\ Cayetano Heredia, \\ Universidad Peruana \\ Cayetano Heredia, Lima, \\ Perú. \\ Correspondencia \\ P. Aguilar-León \\ Hospital Nacional Cayetano \\ Heredia, Universidad \\ Peruana Cayetano Heredia. \\ Av. Honorio Delgado 262, \\ San Martín de Porres 15102, \\ Lima / Lima - 07001, Perú. \\ pool.aguilar@upch.pe
}


la precaria seguridad laboral e informalidad en el país 9,10,11. Los resultados son las consecuencias de una pobre inversión en salud, educación y trabajo que viene de décadas atrás.

Nuestra latente preocupación es la situación posterior a la pandemia de los pacientes diagnosticados con tuberculosis (TB) pulmonar, decimoséptima causa de mortalidad total en el país 12, debido a su importante componente social, asociado a la pobreza y desnutrición, problemas endémicos del Perú. En el 2019 se notificaron 32.970 casos con una tasa de incidencia en 88,6 casos nuevos de TB por cada 100 mil habitantes. Las cifras revelan un repunte en el número de casos notificados con respecto a años previos. Sin embargo, es importante destacar que la identificación de sintomáticos respiratorios, en números absolutos, también se ha incrementado sostenidamente en los últimos 5 años, alcanzando un valor de 2.049.897 sintomáticos respiratorios identificados el año pasado ${ }^{13}$. La incertidumbre se cierne en el impacto de la pandemia sobre el avance sostenido que ha venido presentando el sistema nacional de notificación y vigilancia, y que aunado a la crisis socioeconómica venidera, puede ahondar la problemática sanitaria de la TB en nuestro país.

Los pacientes con TB conllevan un riesgo de sucumbir ante el nuevo coronavirus por su condición de vulnerabilidad a través del daño pulmonar crónico, las comorbilidades asociadas, incluyendo infección por VIH y diabetes mellitus, la desnutrición y la pobreza 14. Sobre lo mencionado, un primer estudio observacional preliminar en China ya identificaba a la TB pulmonar como factor de riesgo para severidad de COVID-19 15. Hallazgos identificados previamente con neumonías virales por Influenza, MERS-CoV y SARS-CoV-1 16,17. Asimismo, ya se vienen reportando series de casos de coinfección que deslizan un diagnóstico de TB posterior a la infección por el nuevo coronavirus 18 . Sin embargo, la alta prevalencia global de la TB y la carga cada vez mayor de COVID-19 denota más probabilidad de que la coinfección sea una ocurrencia coincidente más que una asociación causal 19. Sin embargo, se discuten riesgos adicionales, basados en la desatención hacia el diagnóstico, seguimiento de casos, acceso a tratamiento e investigación para el desarrollo de nuevas drogas, tests diagnósticos y ensayos de vacunas por orientar esfuerzos y recursos a combatir el COVID-19. Los resultados esperados son previsibles e incluyen un aumento en la notificación de casos, transmisión comunitaria y multidrogorresistencia ${ }^{20}$. Dicha población parte con desventaja en la carrera por sobrevivir al nuevo coronavirus.

Asimismo, los sobrevivientes de COVID-19 pueden tener un alto riesgo de tuberculosis, y la infección por el nuevo coronavirus en sí misma podría aumentar el riesgo de progresión de la infección latente a enfermedad activa 21. El mecanismo hipotético yace en la inmunodepresión. La respuesta inmune desregulada inicial, a través del fenómeno denominado tormenta de citoquinas, tiene una etapa de supresión inmune posterior que se caracteriza por una reducción sostenida y sustancial del recuento de linfocitos periféricos, principalmente células T CD4 y CD8 22. El mecanismo de la linfopenia aún es desconocido; pero nuevos estudios hipotetizan sobre la posibilidad de que el nuevo coronavirus pueda infectar directamente a los linfocitos, particularmente las células T, e iniciar o promover la muerte celular, dando lugar a respuestas antivirales dañadas y predisposición a sobreinfección bacteriana 23. Dicha afirmación se basa parcialmente en hallazgos de replicación viral en población linfocitaria en estudios previos con SARS-CoV-1 24.

Mitigar los efectos de la pandemia en el Perú, sin desatender las enfermedades pre-existentes, es un reto para la salud pública nacional. En ese sentido, es una obligación anticipar la sinergia potencialmente destructiva entre COVID-19, TB y la pobreza. Coincidimos sobre el beneficio sanitario del trabajo que los programas de TB han realizado a lo largo de los años en áreas como control de infecciones, diagnóstico, localización de contactos y aislamiento. Existe una clara oportunidad de aprovechar los conocimientos adquiridos para el control de la pandemia 25. Asimismo, cualquier inversión realizada en el manejo del coronavirus debe aprovecharse para la atención de pacientes con TB; citando como ejemplos el aislamiento social efectivo, lavado de manos y uso de mascarilla obligatorio en lugares públicos. De igual manera, la implementación de nuevos laboratorios moleculares deben servir para optimizar y agilizar el diagnóstico de TB en el futuro. Importante también es el desarrollo de estudios longitudinales para identificar las consecuencias futuras de la pandemia. La situación actual está cambiando radicalmente la forma en que manejamos la TB en el futuro inmediato y desenmascarando vulnerabilidades; es necesario retomar el rumbo a fin de evitar una crisis sanitaria sin precedentes. 


\section{Colaboradores}

P. Aguilar-León, J. Cotrina-Castañeda y E. ZavalaFlores participaron en la concepción, revisión crítica relevante del contenido intelectual, redacción del artículo y aprobación final de la versión que se publicará; siendo responsables de todos los aspectos del trabajo.

\section{Informaciones adicionales}

ORCID: Pool Aguilar-León (0000-0001-74026586); Jose Cotrina-Castañeda (0000-0002-81127606); Ernesto Zavala-Flores (0000-0002-73895576).

\section{Referencias}

1. Rodríguez-Morales AJ, Gallego V, Escalera-Antezana JP, Méndez CA, Zambrano LI, FrancoParedes C, et al. COVID-19 in Latin America: the implications of the first confirmed case in Brazil. Travel Med Infect Dis 2020; 35:101613.

2. Dong E, Du H, Gardner L. An interactive webbased dashboard to track COVID-19 in real time. Lancet Infect Dis 2020; 20:533-4.

3. Aquino M, Garrison C. Peru records first confirmed case of coronavirus, President Vizcarra says. Reuters 2020; 6 mar. https://www.reuters. com/article/us-health-coronavirus-peru/perurecords-first-confirmed-case-of-coronaviruspresident-vizcarra-says-idUSKBN20T1S9.

4. Amazonas Explorer. Coronavirus in Peru: the latest updates. https://amazonas-explorer. $\mathrm{com} /$ is-there-coronavirus-in-peru/\#Timeline of_coronavirus_cases_in_Peru (accedido el 20/ Ago/2020).

5. Ministerio de Salud. Sala situacional: COVID-19 Perú. https://covid19.minsa. gob.pe/sala_situacional.asp (accedido el 20/ Ago/2020).

6. Munayco C, Chowell G, Tariq A, Undurraga EA, Mizumoto K. Risk of death by age and gender from COVID-19 in Peru, March-May, 2020. Aging 2020; 12:13869-81.

7. Worldometer. COVID-19 coronavirus pandemic. https://www.worldometers.info/coro navirus/ (accedido el 20/Ago/2020).

8. Burki T. COVID-19 in Latin America. Lancet Infect Dis 2020; 20:547-8.

9. Taj M, Kurmanaev A. El virus exhibe las debilidades de la historia de éxito de Perú. The New York Times 2020; 12 jun. https://www. nytimes.com/es/2020/06/12/espanol/americalatina/peru-coronavirus-corrupcion-muertes. html.

10. Castillo M. Peru seemed to do everything right. So how did it become a Covid-19 hotspot? CNN 2020; 27 may. https://edition. cnn.com/2020/05/25/americas/peru-covidhotspot-why-intl/index.html.

11. Pighi P. Cuarentena en Perú: 5 factores que explican por qué las medidas de confinamiento no impiden que sea el segundo país de América Latina con más casos de covid-19. BBC News 2020; 22 may. https://www.bbc.com/mundo/ noticias-america-latina-52748764.

12. Ministerio de Salud. Principales causas de mortalidad por sexo. Perú - año 2017. https:// www.minsa.gob.pe/reunis/recursos_salud/ MORTG002017.asp (accedido el 20/Ago/ 2020).

13. Dirección de Prevención y Control de la Tuberculosis, Ministerio de Salud. Perfil de la tuberculosis - Perú. http://www.tuberculosis.minsa. gob.pe/DashboardDPCTB/PerfilTB.aspx (accedido el 20/Ago/2020).

14. Abdool Karim Q, Abdool Karim SS. COVID-19 affects HIV and tuberculosis care. Science 2020; 369:366-8. 
15. Liu Y, Bi L, Chen Y, Wang Y, Fleming J, Yu Y, et al. Active or latent tuberculosis increases susceptibility to COVID-19 and disease severity. medRxiv 2020; 16 mar. https://www.medrxiv. org/content/10.1101/2020.03.10.20033795v1.

16. Alfaraj SH, Al-Tawfiq JA, Altuwaijri TA, Memish ZA. Middle East respiratory syndrome coronavirus and pulmonary tuberculosis coinfection: implications for infection control. Intervirology 2017; 60:53-5.

17. Walaza S, Cohen C, Nanoo A, Cohen AL, McAnerney J, von Mollendorf C, et al. Excess mortality associated with influenza among tuberculosis deaths in South Africa, 1999-2009. PLoS One 2015; 10:e129173.

18. Motta I, Centis R, D’Ambrosio L, García-García J-M, Goletti D, Gualano G, et al. Tuberculosis, COVID-19 and migrants: preliminary analysis of deaths occurring in 69 patients from two cohorts. Pulmonology 2020; 26:233-40.

19. Tadolini M, García-García JM, Blanc FX, Borisov S, Goletti D, Motta I, et al. On tuberculosis and COVID-19 co-infection. Eur Respir J 2020; 56:2002328.
20. Togun T, Kampmann B, Stoker NG, Lipman M. Anticipating the impact of the COVID-19 pandemic on TB patients and TB control programmes. Ann Clin Microbiol Antimicrob 2020; 19:21.

21. McQuaid CF, McCreesh N, Read JM, Sumner T; CMMID COVID-19 Working Group; Houben RMGJ, et al. The potential impact of COVID-19-related disruption on tuberculosis burden. Eur Respir J 2020; 56:2001718.

22. Mehta P, McAuley DF, Brown M, Sanchez E, Tattersall RS, Manson JJ, et al. COVID-19: consider cytokine storm syndromes and immunosuppression. Lancet 2020; 395:1033-4.

23. Li H, Liu L, Zhang D, Xu J, Dai H, Tang N, et al. SARS-CoV-2 and viral sepsis: observations and hypotheses. Lancet 2020; 395:1517-20.

24. Gu J, Gong E, Zhang B, Zheng J, Gao Z, Zhong $Y$, et al. Multiple organ infection and the pathogenesis of SARS. J Exp Med 2005; 202:415-24.

25. Saunders MJ, Evans CA. COVID-19, tuberculosis and poverty: preventing a perfect storm. Eur Respir J 2020; 56:2001348. 